\title{
OPEN Scalp HFO rates decrease after successful epilepsy surgery and are not impacted by the skull defect resulting from craniotomy
}

\author{
Dorottya Cserpan ${ }^{1}$, Antonio Gennari ${ }^{1,2}{ }^{2}$ Luca Gaito ${ }^{1,3}$, Santo Pietro Lo Biundo ${ }^{1}$, \\ Ruth Tuura ${ }^{2,4,5}$, Johannes Sarnthein ${ }^{3,4,6}$ \& Georgia Ramantani ${ }^{1,4,5} \bowtie$
}

Epilepsy surgery can achieve seizure freedom in selected pediatric candidates, but reliable postsurgical predictors of seizure freedom are missing. High frequency oscillations (HFO) in scalp EEG are a new and promising biomarker of treatment response. However, it is unclear if the skull defect resulting from craniotomy interferes with HFO detection in postsurgical recordings. We considered 14 children with focal lesional epilepsy who underwent presurgical evaluation, epilepsy surgery, and postsurgical follow-up of $\geq 1$ year. We identified the nearest EEG electrodes to the skull defect in the postsurgical MRI. We applied a previously validated automated HFO detector to determine HFO rates in presurgical and postsurgical EEG. Overall, HFO rates showed a positive correlation with seizure frequency $(p<0.001)$. HFO rates in channels over the HFO area decreased following successful epilepsy surgery, irrespective of their proximity to the skull defect $(p=0.005)$. HFO rates in channels outside the HFO area but near the skull defect showed no increase following surgery $(p=0.091)$ and did not differ from their contralateral channels $(p=0.726)$. Our observations show that the skull defect does not interfere with postsurgical HFO detection. This supports the notion that scalp HFO can predict postsurgical seizure freedom and thus guide therapy management in focal lesional epilepsy.

For children with drug-resistant focal lesional epilepsy, epilepsy surgery is the only treatment that bears the potential of cure, as recently shown in a randomized controlled trial ${ }^{1}$. Overall, seizure freedom is attained in two-thirds of patients, and cure, defined as seizure freedom off anti-seizure medication (ASM), is achieved in one-third ${ }^{2-8}$. Following surgery, the lack of residual lesions in postsurgical MRI and the lack of epileptic discharges in postsurgical EEG have been suggested as potential predictors of seizure freedom in pediatric cohorts $^{2-6}$, albeit with contradictory results between studies. Considering that pediatric lesional epilepsies are not necessarily MRI-positive ${ }^{3,5,6}$ and that the relationship of the lesion with the epileptogenic zone is not necessarily straightforward ${ }^{9}$, EEG biomarkers appear more suitable for the measurement of residual epileptogenicity and, thus, for the prediction of seizure outcome. Predicting seizure freedom following surgery with confidence is crucial for therapy management and specifically for the timely initiation of ASM withdrawal. Early ASM withdrawal after successful epilepsy surgery determines postsurgical cognitive gains ${ }^{10,11}$, as the only modifiable postsurgical predictor of optimal cognitive outcome ${ }^{12}$.

High frequency oscillations (HFO) in scalp EEG are a new and promising non-invasive epilepsy biomarker providing added prognostic value, particularly in the pediatric population ${ }^{13}$. Originally, scalp HFO have been associated with the seizure onset zone in drug-resistant focal epilepsy ${ }^{14-21}$, thus delineating surgical resection. Currently, scalp HFO are investigated as biomarkers of seizure propensity, disease severity, and treatment response $^{13}$. In our recent study, scalp HFO rates in pediatric drug-resistant focal lesional epilepsy showed a positive correlation with seizure frequency and decreased with seizure cessation following successful epilepsy surgery $^{14}$. This observation underlined the potential of scalp HFO as a widely accessible non-invasive biomarker of treatment response, potentially facilitating outcome prognostication. However, comparisons between

\footnotetext{
${ }^{1}$ Department of Neuropediatrics, University Children's Hospital Zurich, Steinwiesstrasse 75, 8032 Zurich, Switzerland. ${ }^{2}$ MR-Research Centre, University Children's Hospital Zurich, Zurich, Switzerland. ${ }^{3}$ Department of Neurosurgery, University Hospital Zurich, Zurich, Switzerland. ${ }^{4}$ University of Zurich, Zurich, Switzerland. ${ }^{5} \mathrm{Children's}$ Research Centre, University Children's Hospital Zurich, Zurich, Switzerland. ${ }^{6} K$ linisches Neurozentrum Zürich, University Hospital Zurich, Zurich, Switzerland. ${ }^{\circledR}$ email: georgia.ramantani@kispi.uzh.ch
} 
consecutive scalp EEG recordings before and after epilepsy surgery are only valid under the assumption that the craniotomy with the resulting skull defect has no significant impact on scalp HFO detection.

In scalp EEG, the major resistive element is the bone with a resistance of around $40,000 \Omega$ for $1 \mathrm{~cm}^{2}$ of skull, in contrast to $12,000 \Omega$ for $1 \mathrm{~cm}^{2}$ of dura mater, and $1000 \Omega$ for $1 \mathrm{~cm}^{2}$ of scalp in adults. EEG signals passing through the intact skull are subject to the averaging and smoothing effects of the bone ${ }^{22,23}$ that skull defects due to craniotomy can partially abolish ${ }^{24,25}$. Signal attenuation and smoothing with bone present and signal accentuation and deblurring with bone absent are both frequency-dependent, i.e., the faster the EEG rhythm, the more pronounced the impact on the signal ${ }^{24,25}$. Therefore, EEG recorded over a skull defect is expected to show higher overall spectral power and a greater proportion of fast components due to decreased high frequency attenuation compared to EEG from a homologous head region with an intact skull, corresponding to a "breach rhythm" 26 . The question remains whether a skull defect following epilepsy surgery can interfere with the detection and interpretation of scalp EEG signals in the HFO range and thus with the validity of scalp HFO as a biomarker of treatment response.

To assess the effect of skull defects resulting from craniotomy on the validity of postsurgical scalp HFO as a biomarker of treatment response, we retrospectively analyzed scalp EEG from children and adolescents with drug-resistant focal lesional epilepsy before and after resective surgery that involved craniotomy. To address the hypothesis that scalp HFO rates decrease over the HFO area following successful epilepsy surgery, irrespective of the distance to the skull defect resulting from craniotomy, we investigated pre- to postsurgical changes in HFO rates depending on the postsurgical seizure outcome and the distance to the skull defect. To address the hypothesis that scalp HFO rates outside the HFO area but close to the skull defect resulting from craniotomy show no increase following surgery, we investigated pre- to postsurgical changes in scalp HFO rates depending on the distance from the skull defect.

\begin{abstract}
Methods
Patient recruitment. We prospectively enrolled 37 consecutive children and adolescents with drug-resistant focal lesional epilepsy who underwent presurgical evaluation, resective epilepsy surgery between July 2017 and November 2020, and postsurgical follow-up at the University Children's Hospital Zurich, according to our institutional protocol. For the current study focusing on the effect of the skull defect resulting from craniotomy on scalp HFO rates, we considered those patients for further analysis that fulfilled the following inclusion criteria: (1) presurgical and postsurgical scalp EEG recorded at a high sampling frequency $(>1000 \mathrm{~Hz})$, (2) containing at least $10 \mathrm{~min}$ of NREM sleep, and (3) recorded at $>2 \mathrm{~h}$ from the most recent seizure, and (4) followup $\geq 1$ year after epilepsy surgery.

Presurgical evaluation at $\leq 3$ months before surgery included scalp EEG and high-resolution brain MRI, while postsurgical evaluation at three months after surgery included scalp EEG and MRI according to the same protocol. Epilepsy substrates were determined by histopathology. Postsurgical seizure outcome was depicted according to the Engel scale ${ }^{27}$. Seizure frequency was assessed by long-term video-EEG or seizure diaries at the time of the presurgical and postsurgical scalp EEG.

The collection of patient data and the scientific analysis were approved by and performed according to the guidelines and regulations of the local ethics committee (Kantonale Ethikkommission Zürich, KEK-ZH PB-2021-01246). All parents and patients, where applicable, have given written informed consent.
\end{abstract}

Electrode positions in relation to the skull defect resulting from craniotomy. Postsurgical MRI scans were performed on a 3-Tesla scanner (Discovery $750^{\circ}$, General Electric Medical System, Milwaukee, WI, USA) with an 8-channel head coil, including a volumetric, whole-brain, T1-weighted sequence (3D-FSPGRBRAVO sequence; matrix size, $512 \times 512$ with 300 contiguous slices; field of view: $256 \mathrm{~mm}$; isotropic resolution, $1 \mathrm{~mm}$; sagittal slice orientation; repetition time: $12.1 \mathrm{~ms}$; echo time: $5.2 \mathrm{~ms}$; flip angle: $12^{\circ}$; inversion time: $450 \mathrm{~ms}$ ). The 3D T1-weighted DICOM images were converted to nifti files using dcm2nii and then loaded into the 3D Slicer (version 4.10). The craniotomy site was identified by an experienced radiologist (A.G.), and the bone flap was manually segmented, using post-processing tools (e.g., windowing and magnification) and switching between image plane orientations. The following smoothing filters were then applied to the generated files: (1) closing, which filled sharp corners and holes in the segmentation, (2) opening, which removed extrusion, and (3) joint smoothly, which evened the segmentation. The resulting images were re-evaluated and adjusted. Finally, the bone flap volumes were calculated, approximating the segmentation areas.

Native nifti files were reloaded into the Geodesic Slicer module 3D Slicer. Geodesic Slicer enables the creation of a triangular mesh morphed on the patient's head, reconstructed from the T1-weighted images. The mesh is modeled according to the geodesic distance measurement, defined as the shortest path between two points in a curved space. After placing four reference points (fiducials) on the nasion, the inion, and the left and right tragi (i.e., anatomical landmarks), the electrode locations of the 10-20 EEG system were automatically generated. The bone flap segmentations were loaded into 3D Slicer, and the distance between the skull defect resulting from craniotomy (skull incision) and the closest electrodes was automatically calculated using classical Euclidean distance formulas for all segmentation borders. For visualization, we used the FieldTrip package (Matlab R2020a).

Scalp EEG recording and data selection. Patients underwent afternoon nap $(\mathrm{N}=18)$ or whole-night video-EEG $(\mathrm{N}=10)$ with 21 electrodes placed according to the international 10-20 system. Impedances were typically $\leq 5 \mathrm{k} \Omega$. Recordings were performed at a $1024 \mathrm{~Hz}$ sampling rate by the Deltamed ${ }^{\oplus}$ (Paris, France) EEG system for afternoon nap recordings or the Micromed $^{\circledR}$ (Mogliano Veneto, Treviso, Italy) EEG system for whole night recordings. Sleep stages were marked by experienced neurophysiologists according to the American Academy of Sleep Medicine (AASM) criteria ${ }^{28}$. We considered the NREM sleep stage N3 for further analysis. 
We divided the selected data into 5-min intervals for further processing. During visual pre-processing, 5-min EEG intervals heavily contaminated by artifacts and channels with continuous interference were fully excluded from further analysis. Other artifacts were detected and excluded during the automated HFO detection.

HFO detection and analysis were performed blinded to clinical features of the patients, and the results from $\mathrm{HFO}$ analysis were not considered for clinical decision making.

Automated scalp HFO detection in EEG. To capture the HFO activity with the highest possible spatial resolution, we re-referenced to a bipolar montage using all combinations of neighboring electrodes, thus obtaining 52 bipolar channels ${ }^{29}$.

Scalp HFO detection was conducted with a clinically validated, automated HFO detector ${ }^{14,29-32}$ that operates in three stages ${ }^{32}$. The detector uses Stockwell's algorithm for the time-frequency transform of the EEG signal ${ }^{30}$. In Stage I, baseline detection, a baseline amplitude threshold is determined in artifact-free intervals, selecting epochs of high Stockwell entropy in the ripple band $(80-250 \mathrm{~Hz})$. Events exceeding this threshold are marked as events of interest (EoI). In Stage II, HFO validation by Stockwell transform, the detector selects those EoI where a high-frequency peak is isolated from the low-frequency activity and marks them as HFO. This step reflects the assumption that HFO are brief events with a distinct high-frequency contribution that stands out from the baseline $^{30}$. Finally, in Stage III, artifact rejection, the detector screens EoI selected in stage II to further eliminate artifacts. This step is guided by the assumption that HFO are generated by highly localized cortical tissue and should thus not be detectable across hemispheres ${ }^{33}$. Thus, the detector rejects all EoI co-occurring in homologous channels of the two hemispheres, in addition to those with peak-to-peak amplitude $\geq 40 \mu \mathrm{V}$ or signal-to-noise ratio $<9$. In addition, a z-score is calculated from the $250-500 \mathrm{~Hz}$ band-pass-filtered data over time, and events that occur at high $\mathrm{z}$-score timepoints, exceeding the median $\mathrm{z}$-score by 1.5 times the interquartile range for each patient, are rejected. An exemplary HFO event is shown in Supplementary Fig. S1.

The HFO detector was thus applied to each bipolar channel within each selected 5-min data interval. We calculated the HFO rate for each bipolar channel by dividing the number of detected HFO on each channel by the duration of the analyzed EEG recording, resulting in the unit $\mathrm{HFO} / \mathrm{min}$.

We controlled for the clinical plausibility of scalp HFO rate distributions by comparing the HFO area with the localization of the presumed epileptogenic zone, as defined by presurgical evaluation, and with the localization of the consequent resection.

There was neither visual marking nor manual rejection of artifacts, rendering the HFO detection fully automated and prospective.

Channels of interest. HFO area channels were defined by consistently high HFO rates across scalp EEG channels for each patient ${ }^{31}$. First, we calculated the rate threshold $\left(97.5^{\text {th }}\right.$ percentile of the HFO rate distribution) from the presurgical EEG across data intervals and channels. Then, we counted the number of intervals with an above-threshold HFO rate for each channel. Finally, we calculated the $97.5^{\text {th }}$ percentile of these occurrence values. Channels with higher occurrence values constituted the HFO area.

Skull defect channels were defined as channels including electrodes located at $\leq 1 \mathrm{~cm}$ distance from the skull defect resulting from craniotomy (skull defect electrodes, Fig. 1). For example, assuming that the C3 electrode was located at a distance of $0.3 \mathrm{~cm}$ from the skull defect, the channels F3-C3, C3-P3, T5-C3, C3-Pz, C3-Fz would be considered skull defect channels.

Contralateral skull defect channels were those contralateral to skull defect channels.

Statistics. To evaluate scalp HFO rates as an epilepsy biomarker, we compared the HFO rates within the $\mathrm{HFO}$ area with seizure frequency as a measure of disease severity ${ }^{14}$.

We then established a classification threshold using the optimal operating point of the receiver operating characteristic (ROC) curve, as in our previous work ${ }^{14}$. In addition, we challenged the predictive performance of the HFO threshold rate methodology by applying two-fold cross-validation. We randomly shuffled the data array and split it into two equal size datasets $\mathrm{d}_{0}$ and $\mathrm{d}_{1}$, each containing 14 scalp EEG recordings. We then trained on $\mathrm{d}_{0}$ and validated on $\mathrm{d}_{1}$, followed by training on $\mathrm{d}_{1}$ and validating on $\mathrm{d}_{0}$, then repeating this procedure two times the number of recordings. We defined as true positive (TP) a scalp EEG showing equal or above-threshold HFO rates in patients with active epilepsy (all patients before surgery, patients with Engel II-IV outcome after surgery). We defined as false positive (FP) a scalp EEG showing equal or above-threshold HFO rates in seizure-free patients after surgery (Engel I). We defined as false negative (FN) a scalp EEG showing below-threshold HFO rates in patients with seizure recurrence after surgery (Engel II-IV). We defined as true negative (TN) a scalp EEG showing below-threshold HFO rates in seizure-free patients after surgery (Engel I). The positive predictive value $(\mathrm{PPV})$ of scalp HFO rates for active epilepsy was calculated as $\mathrm{PPV}=\mathrm{TP} /(\mathrm{TP}+\mathrm{FP})$, the negative predictive value $(\mathrm{NPV})$ as $\mathrm{NPV}=\mathrm{TN} /(\mathrm{TN}+\mathrm{FN})$, and the accuracy $=(\mathrm{TP}+\mathrm{TN}) / \mathrm{N}$, with $\mathrm{N}$ signifying the total number of analyzed EEG recordings.

To investigate the impact of the skull defect on HFO detection, we compared HFO rates and amplitudes averaged over the channels of interest and described distributions by their median and interquartile range (iqr). To characterize the effect of the skull defect resulting from craniotomy on HFO rates, we used the non-parametric Wilcoxon signed-rank test for comparisons of these values in presurgical to postsurgical EEG or between ipsilateral and contralateral channels in the postsurgical EEG for each patient. We used the Spearman's rank correlation to assess the relationship between the seizure frequency and the mean HFO rates within the HFO area.

Statistical analysis was performed with Matlab R2020a. It should be noted that the MATLAB signed-rank function applied in our study handles zero-inflation by considering only non-zero values. Significance was established at $p<0.05$. We did not perform adjustment for multiple comparisons. 


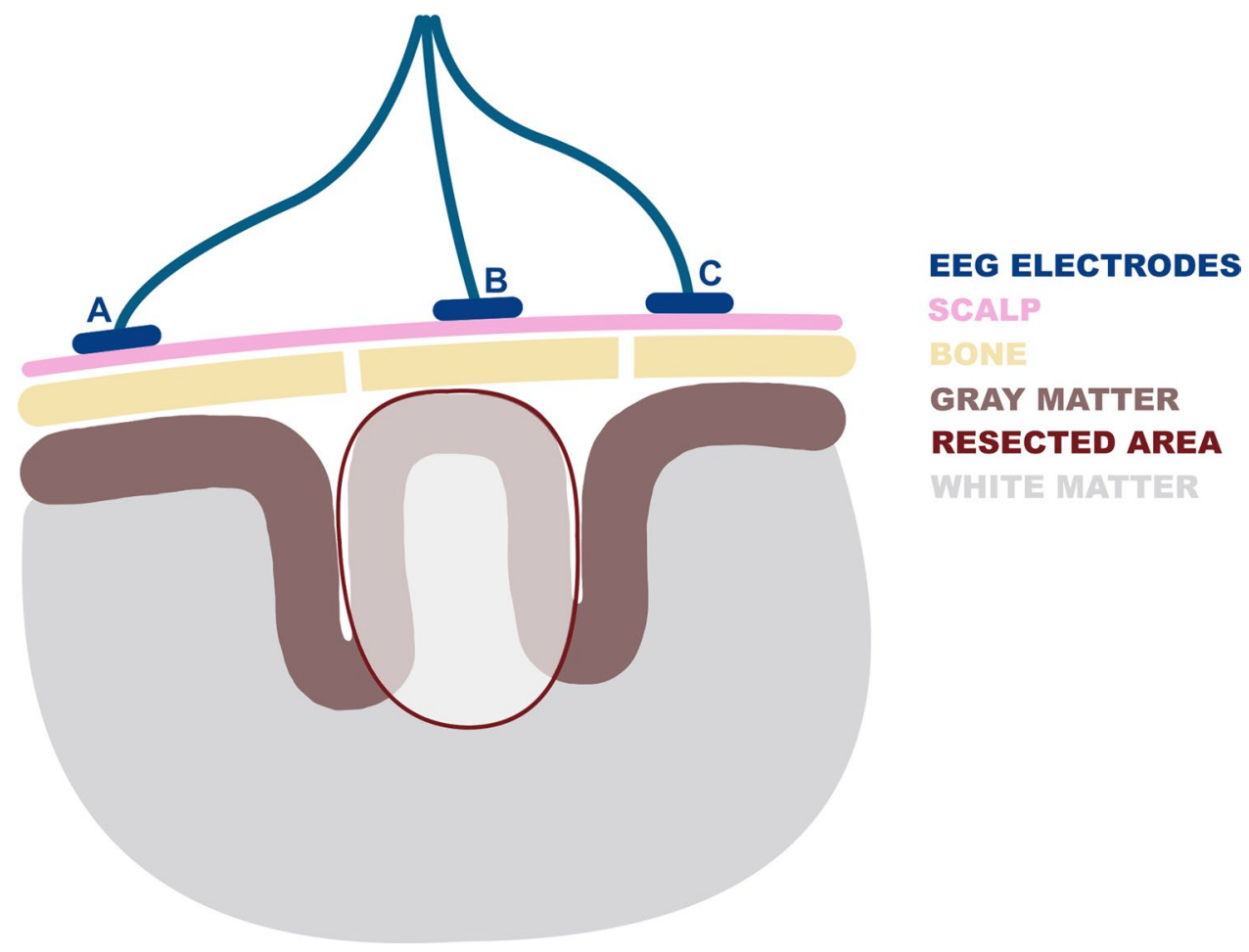

Figure 1. Scalp EEG recording setup following epilepsy surgery. Position of the 10-20 system EEG electrodes following epilepsy surgery in relation to the skull defect resulting from craniotomy and the resected area. For illustrative purposes, we consider that the presurgically defined HFO area overlaps with the resected area depicted here. Electrodes A, B, and C are all located ipsilateral to the craniotomy. Electrode A is located $>1 \mathrm{~cm}$ from the skull defect, the HFO area, and the resection area, and thus does not correspond to a channel of interest. Electrode B is located within the HFO area and is thus involved in HFO area channels. Electrode C is located outside the HFO and the resection area but close to the skull defect $(\leq 1 \mathrm{~cm})$ and is thus involved in skull defect channels.

\section{Results}

Patient characteristics and HFO rates. We included 14 patients ( 10 female) with mean age 8.9 years at surgery (range 1.5-16.5 years) that fulfilled all inclusion criteria. Etiology included cortical malformations and benign tumors in six patients each, cavernomas in two patients, and one patient with Sturge Weber syndrome. The lateralization of the epileptogenic zone and surgical resection was left in eight patients, and the localization was frontal in six patients, temporal in three, parietal in two, and temporo-parieto-occipital in the remaining three patients. Ten of $14(71 \%)$ patients were seizure-free (Engel Ia) at the last follow-up (mean 2.2 years, range 1.0-4.8 years) following epilepsy surgery, whereas six were seizure-free off ASM (four at $\geq 2$-year follow-up). Overall, the median seizure frequency dropped from 20 seizures/month (range 20-1290) at the presurgical EEG to 0 seizures/month (range $0-240$ ) at the postsurgical EEG.

We analyzed $750 \mathrm{~min}$ of presurgical and postsurgical EEG data, including $120 \mathrm{~min}$ of N2 and $630 \mathrm{~min}$ of N3 sleep. In total, we detected $2200 \mathrm{HFO}$, including 563 in N2 and 1637 in N3 sleep. The median duration of analyzed data per patient was 50 min (iqr 30) with a median of 85 (iqr 168) detected HFO per patient. The localization of the HFO area matched on a lobar level the epileptogenic zone, as determined by the electroclinical correlations and the presence of focal MRI lesions in all patients. Following resective surgery through craniotomy, five patients each had one or two skull defect electrodes, one patient had three skull defect electrodes, and three patients had no skull defect electrodes.

The clinical features of our patients, including the location of the skull defect electrodes, are given in Table 1.

HFO rates over the HFO area decrease after successful epilepsy surgery. In our cohort, scalp $\mathrm{HFO}$ rates in HFO area channels correlated with seizure frequency (Spearman's rho 0.740, $p<0.001$ ). Scalp HFO rates in HFO area channels exceeded the threshold of $0.124 \mathrm{HFO} / \mathrm{min}$ based on the ROC curve (Supplementary Fig. S2a) in 17 EEG recordings, all from patients with active epilepsy (PPV=100\%) and fell below this threshold in 11 recordings, ten from patients that achieved postsurgical seizure freedom (NPV $=91 \%$, accuracy $=96 \%$ ). The two-fold cross-validation supported the validity of our findings (median PPV $=100 \%$, iqr $0 \%$; median NPV $=83 \%$, iqr $29 \%$; median accuracy $=93 \%$, iqr $7 \%$ ), while the median HFO rate threshold matched the threshold deriving from the full dataset (median 0.124, iqr 0.001: Supplementary Fig. S2b). Scalp HFO rates 


\begin{tabular}{|c|c|c|c|c|c|c|c|c|}
\hline Pat. Nr & Sex & Etiology & Age at surgery, years & Resected area & $\begin{array}{l}\text { Skull defect } \\
\text { electrodes }\end{array}$ & $\begin{array}{l}\text { Follow-up duration, } \\
\text { years }\end{array}$ & $\begin{array}{l}\text { Seizure outcome } \\
\text { (Engel) }\end{array}$ & ASM withdrawn \\
\hline 1 & f & Ganglioglioma & 1.5 & R temporal & $\mathrm{T} 4$ & 1.5 & IV & No \\
\hline 2 & f & Low-grade glioma & 2.5 & L frontal & None & 1.7 & Ia & No \\
\hline 3 & f & FCD 2b & 2.8 & $\mathrm{R}$ frontal & $\mathrm{Fz}$ & 1.7 & IV & No \\
\hline 4 & f & MOGHE & 3.6 & $\mathrm{~L}$ frontal & Fz, F3, C3 & 1.6 & Ia & No \\
\hline 5 & f & $\begin{array}{l}\text { Sturge Weber syn- } \\
\text { drome }\end{array}$ & 3.8 & R temporo-occipital & $\mathrm{O} 2, \mathrm{P} 4$ & 4.2 & Ia & No \\
\hline 6 & f & FCD 2a & 5.3 & $\mathrm{R}$ frontal & $\mathrm{C} 4$ & 1.3 & IV & No \\
\hline 7 & $\mathrm{~m}$ & FCD la & 7.8 & $\begin{array}{l}\text { L temporo-parieto- } \\
\text { occipital }\end{array}$ & T3, F7 & 4.8 & Ia & No \\
\hline 8 & $\mathrm{~m}$ & Ganglioglioma & 10.5 & L temporal & T5 & 1.8 & Ia & Yes \\
\hline 9 & f & Angiocentric glioma & 11.4 & R parietal & $\mathrm{Oz}, \mathrm{Pz}$ & 1.0 & Ia & Yes \\
\hline 10 & $\mathrm{~m}$ & FCD IIa & 13.0 & L parietal & T3, F7 & 1.0 & IV & No \\
\hline 11 & $\mathrm{~m}$ & Cavernoma & 13.1 & L frontal & None & 2.8 & Ia & Yes \\
\hline 12 & f & Ganglioglioma & 16.0 & L temporal & T3, F7 & 2.7 & Ia & Yes \\
\hline 13 & f & Cavernoma & 16.3 & L frontal & None & 2.5 & Ia & Yes \\
\hline 14 & f & $\begin{array}{l}\text { FCD IIIb, gangli- } \\
\text { oglioma }\end{array}$ & 16.5 & R temporo-parietal & T6 & 2.0 & Ia & Yes \\
\hline
\end{tabular}

Table 1. Clinical features of our patients. Clinical features include the etiological substrate, as verified by histopathology, the lobar localization and lateralization of the resected area, the skull defect electrodes in postsurgical EEG, the postsurgical seizure outcome, and the ASM status at last follow-up for each patient. Pat. Patient, $n r$ number, $m$ male, $f$ female, FCD focal cortical dysplasia, MOGHE mild malformation of cortical development with oligodendroglial hyperplasia and epilepsy, $L$ left, $R$ right, $A S M$ anti-seizure medication.

a Presurgical scalp EEG recording

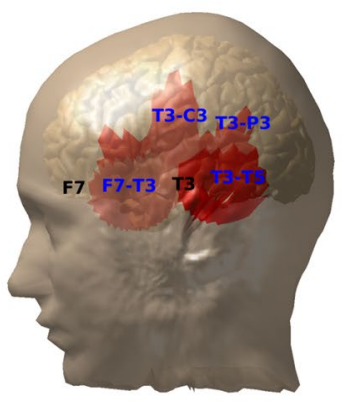

\section{b Resective surgery through craniotomy}

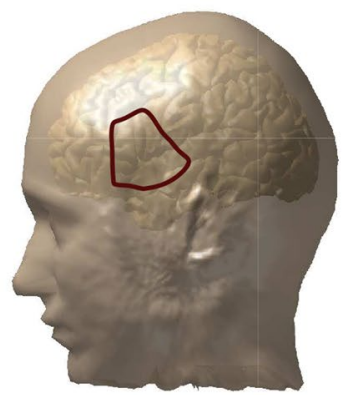

\section{c Postsurgical scalp EEG recording}

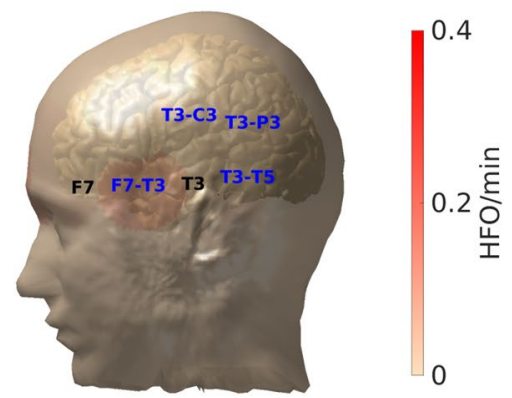

Figure 2. Scalp HFO rate distribution in presurgical and postsurgical EEG in relation to the skull defect resulting from craniotomy. 3D reconstruction of the skull and the cortical surface generated using the presurgical and postsurgical MRI of patient 12, who had a left temporal ganglioglioma. The spatial distribution of HFO rates in presurgical and postsurgical EEG is depicted using areas marked with different shades of red. Different color intensity corresponds to different HFO rates. HFO area channels are shown in blue, skull defect electrodes in black. (a) In the presurgical scalp EEG, high HFO rates were measured in left temporal channels, matching the location of the HFO area, the epileptogenic zone, and the lesion in the anterior portion of the fusiform gyrus. (b) Given the lesion location, a pterional craniotomy approach (outlined in brown) was chosen to fully resect the epileptogenic zone and lesion. (c) In the postsurgical EEG, HFO rates drastically decreased, in line with postsurgical seizure freedom.

over the HFO area decreased following successful epilepsy surgery, as illustrated in Fig. 2 for the exemplary case of patient 12 .

In the patients in whom postsurgical seizure freedom was achieved (Engel I), scalp HFO rates in HFO area channels were significantly decreased after surgery (Fig. 3), irrespective of their proximity to the skull defect resulting from craniotomy (Supplementary Table S1), whereas no significant change was established in the patients in whom postsurgical seizure freedom was not achieved. Furthermore, in patients in whom postsurgical seizure freedom was achieved, the median scalp HFO rate decreased from $0.287 \mathrm{HFO} / \mathrm{min}$ (iqr 0.107 ) in the presurgical EEG to $0.015 \mathrm{HFO} / \mathrm{min}$ (iqr 0.029) in the postsurgical EEG (Wilcoxon signed-rank, $p=0.005$, $\mathrm{z}=2.803$ ). In contrast, in the patients in whom postsurgical seizure freedom was not achieved (Engel II-IV), the median scalp HFO rate did not differ significantly between the presurgical EEG $(0.514 \mathrm{HFO} / \mathrm{min}$, iqr 0.383$)$ and the postsurgical EEG $(0.506 \mathrm{HFO} / \mathrm{min}$, iqr 0.461; Wilcoxon signed-rank, $p=0.465, \mathrm{z}=0.730)$. 
a HFO rates correlate with seizure frequency

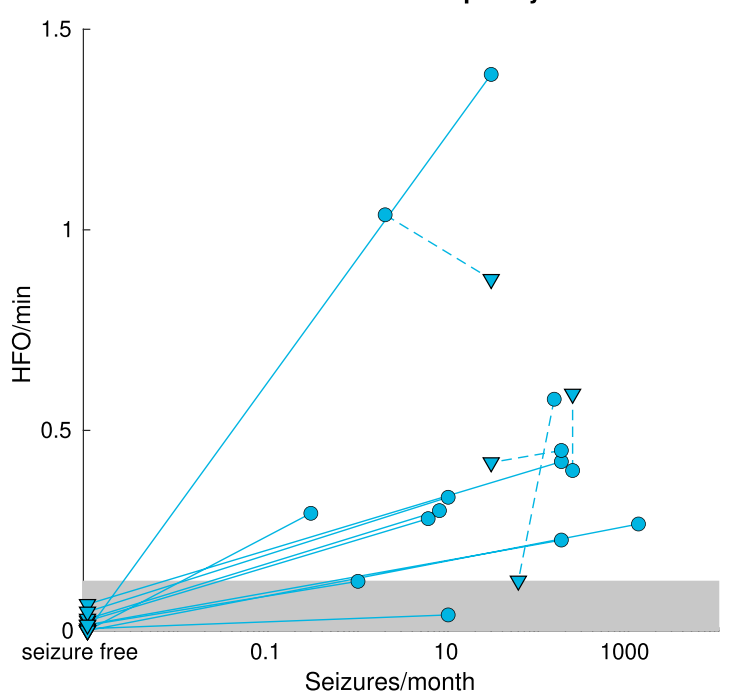

b HFO rates decrease after successful surgery

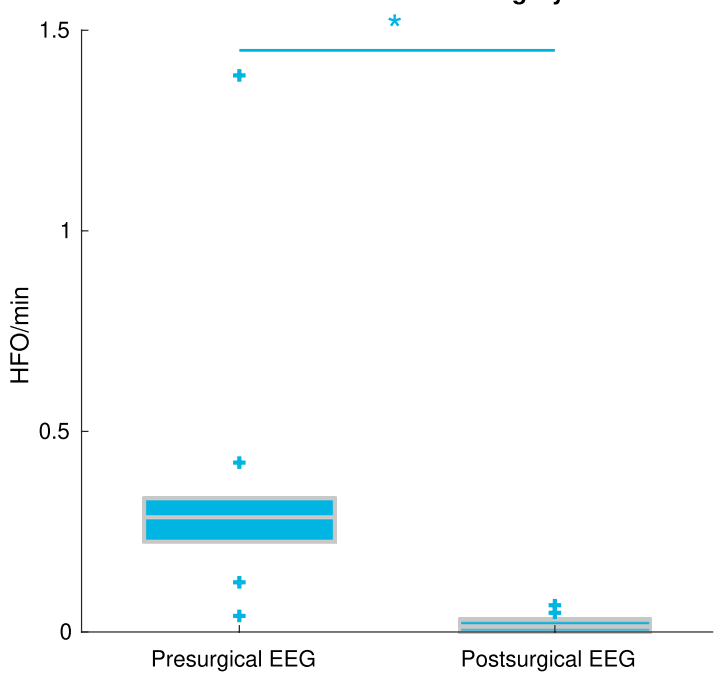

Figure 3. Scalp HFO rates correlate with seizure frequency and decrease after successful epilepsy surgery. (a) Scalp HFO rates in HFO area channels correlate with seizure frequency (Spearman's rho 0.740, $p<0.001$ ). Presurgical HFO rates are indicated by circles and postsurgical HFO rates by triangles. Pre- and postsurgical HFO rates are connected by a solid line for the patients for whom postsurgical seizure freedom was achieved (Engel I) and by a dotted line for patients for whom postsurgical seizure freedom was not achieved (Engel II-IV). The gray area on the bottom of the graph represents the HFO range corresponding to seizure freedom, based on the $0.124 \mathrm{HFO} / \mathrm{min}$ threshold, as established by the ROC curve. 17 of 28 scalp EEG recordings were classified correctly as true positive, ten as true negative, and only one was a false negative. (b) Median scalp HFO rates in HFO area channels decrease after surgery in the patients for whom postsurgical seizure freedom was achieved (Wilcoxon signed-rank: $p=0.005, \mathrm{z}=2.803$ ) from $0.287 \mathrm{HFO} / \mathrm{min}$ (iqr 0.107 ) in the presurgical to $0.015 \mathrm{HFO} / \mathrm{min}$ (iqr 0.029) in the postsurgical EEG.

HFO rates near the skull defect do not increase after epilepsy surgery. In the patients in whom postsurgical seizure freedom was achieved (Engel I), mean HFO rates in skull defect channels within the HFO area decreased significantly (Wilcoxon signed-rank: $p=0.043, \mathrm{z}=2.023$ ) from the presurgical (median 0.280 $\mathrm{HFO} / \mathrm{min}$, iqr 0.491 ) to the postsurgical EEG (median of $0.000 \mathrm{HFO} / \mathrm{min}$, iqr 0.025 ) (Fig. $4 \mathrm{a}$ ). In addition, mean HFO rates in skull defect channels outside the HFO area did not differ significantly (Wilcoxon signedrank: $p=0.091, \mathrm{z}=1.690$ ) between the presurgical (median $0.042 \mathrm{HFO} / \mathrm{min}$, iqr 0.118 ) and the postsurgical EEG (median $0.000 \mathrm{HFO} / \mathrm{min}$, iqr 0.005) (Fig. 4b). Finally, irrespective of the postsurgical seizure outcome, mean $\mathrm{HFO}$ rates in skull defect channels outside the $\mathrm{HFO}$ area (median $0.003 \mathrm{HFO} / \mathrm{min}$, iqr 0.063 ) did not differ significantly (Wilcoxon signed-rank: $p=0.726, \mathrm{z}=0.350$ ) from their contralateral channels (median $0.003 \mathrm{HFO} /$ min, iqr 0.011) in the postsurgical EEG (Fig. 4c).

\section{Discussion}

To our knowledge, the present study is the first to address the impact of skull defects resulting from craniotomy on scalp HFO, by evaluating alterations in HFO rates in relation to the presurgically defined HFO area, the resected area, and the postsurgical skull defect in presurgical and postsurgical EEG recordings from children and adolescents with drug-resistant focal lesional epilepsy. The results validate our earlier observation, deriving from a smaller exploratory cohort recorded with a custom-made amplifier, that HFO rates over the HFO area decrease after successful epilepsy surgery, thus reflecting treatment response, in a larger independent cohort recorded with a commercial device and followed-up considerably longer than our initial cohort ${ }^{14}$. In addition, in the current study we demonstrate for the first time that HFO rates both within and outside the HFO area remain largely unaffected by the skull defect resulting from craniotomy, thus excluding a confounding effect of the skull defect on HFO detection and confirming the validity of comparisons between consecutive EEG recordings. Our study thus serves to establish the utility of scalp HFO in evaluating treatment response, prognosticating seizure outcome, and dictating treatment management following pediatric epilepsy surgery.

Scalp HFO rates decrease after successful epilepsy surgery. In our study, scalp HFO rates over the HFO area decreased following successful epilepsy surgery. These observations corroborate our previous findings, derived from an exploratory dataset recorded by a dedicated 8-channel low-noise amplifier ${ }^{14}$, connected to four adjacent electrodes over the presumed epileptogenic zone and to their contralateral electrodes, with an independent validation dataset recorded by a commercial device using the full 10-20 electrode array, without the requirement of a clear hypothesis regarding the localization of the epileptogenic zone. The previous dataset included a smaller cohort with a mean postsurgical follow-up of 1.2 years, while half of the patients had a followup of $<6$ months. In contrast, the present results were derived from a larger cohort with a postsurgical follow-up 
a Skull defect channels within the HFO area in pre- vs. postsurgical EEG

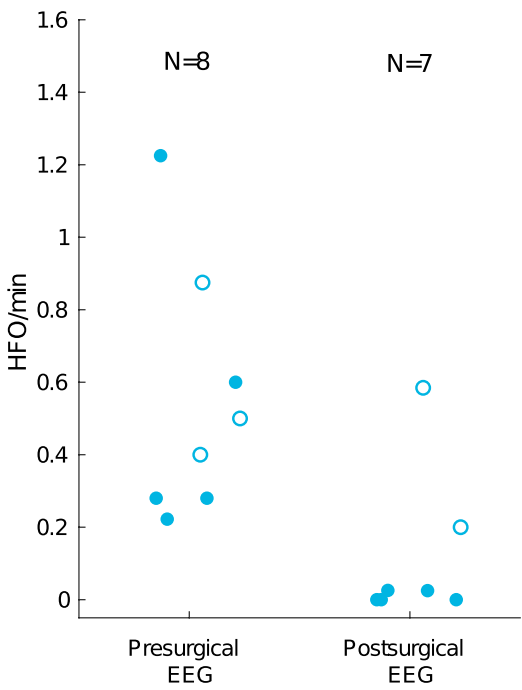

b Skull defect channels outside the HFO area in pre- vs. postsurgical EEG

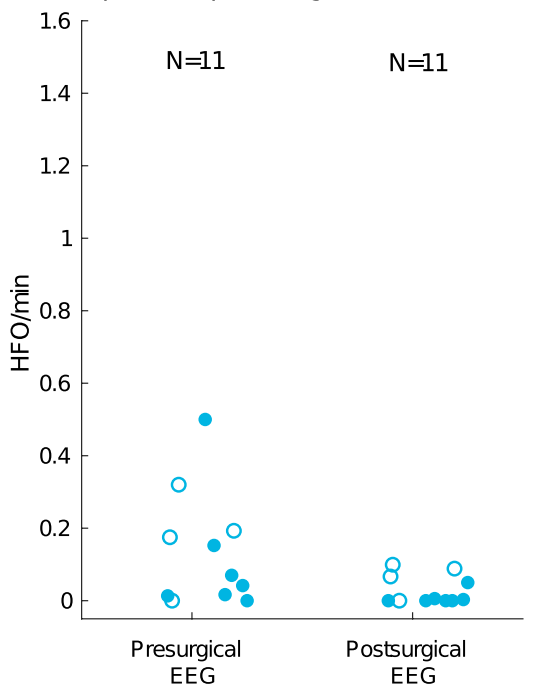

c Skull defect channels outside the HFO area in only postsurgical EEG

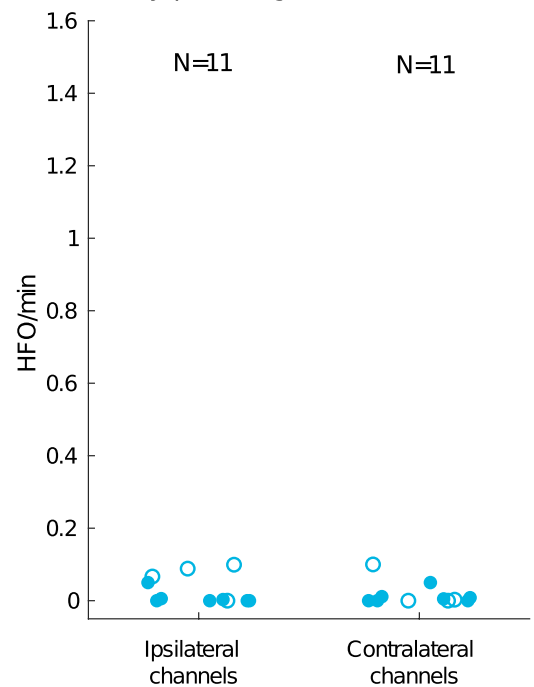

Figure 4. Scalp HFO rates of skull defect channels and their contralateral channels in presurgical and postsurgical EEG recordings. The patients for whom postsurgical seizure freedom was achieved (Engel I) are indicated by blue (full) circles, while patients with postsurgical seizure recurrence (Engel II-IV) are indicated by white (empty) circles. Of the 11 patients with skull defect channels, seven achieved postsurgical seizure freedom and four had postsurgical seizure recurrence. (a) Mean scalp HFO rates of skull defect channels within the HFO area significantly decreased (Wilcoxon signed-rank: $p=0.043, \mathrm{z}=2.023$ ) from the presurgical (median 0.280 $\mathrm{HFO} / \mathrm{min}$, iqr 0.491 ) to the postsurgical EEG (median of $0.000 \mathrm{HFO} / \mathrm{min}$, iqr 0.025 ) in the five patients for whom postsurgical seizure freedom was achieved. (b) Mean scalp HFO rates of skull defect channels outside the HFO area did not differ significantly (Wilcoxon signed-rank: $p=0.091, \mathrm{z}=1.690$ ) between the presurgical (median $0.042 \mathrm{HFO} / \mathrm{min}$, iqr 0.118) and postsurgical EEG (median $0.000 \mathrm{HFO} / \mathrm{min}$, iqr 0.005) in the seven patients for whom postsurgical seizure freedom was achieved. (c) Mean HFO rates of skull defect channels outside the HFO area (median $0.003 \mathrm{HFO} / \mathrm{min}$, iqr 0.063 ) did not differ significantly (Wilcoxon signed-rank: $p=0.726, \mathrm{z}=0.350$ ) from their contralateral channels (median $0.003 \mathrm{HFO} / \mathrm{min}$, iqr 0.011 ) in the postsurgical EEG of all patients, regardless of postsurgical seizure outcome.

of 2.2 years, while half of the patients had a follow-up of $>2$ years. Our observations thus support the feasibility, applicability, and validity of our approach in a real-world pediatric setting, drawing from scalp EEG performed in clinical routine, and establish the potential of scalp HFO as a biomarker of treatment response in children and adolescents with focal lesional epilepsy undergoing epilepsy surgery.

Recent studies have supported a link between increased scalp HFO rates and the epileptogenic zone in focal lesional epilepsy in adult cohorts ${ }^{15,16,18,34,35}$, or between decreased scalp HFO rates and treatment response in various epilepsy syndromes ${ }^{36-39}$ in pediatric cohorts. However, data on the response to treatment of scalp HFO related to a focal lesion in pediatric cohorts is scarce, limited to a single study that followed the evolution of HFO rates over consecutive EEG recordings before, during, and after epilepsy surgery ${ }^{14}$. In addition to establishing a link between HFO in the intraoperative ECoG and HFO in the scalp EEG, that study ${ }^{14}$ showed for the first time that the resection of the cortical HFO generators results in a reduction of HFO rates recorded in scalp EEG, paving the way for our current work. However, these preliminary observations were derived from only eight patients with consecutive presurgical and postsurgical scalp EEG recordings, of whom only half had a postsurgical follow-up of over a year. In the present study, the decrease of scalp HFO rates at the 3-month follow-up corresponded to seizure freedom 2.5 years after surgery and after ASM withdrawal in over half of patients, thus supporting the validity and generalizability of our results. Scalp HFO rates also dropped with decreasing seizure frequency in our pediatric focal lesional epilepsy cohort, thus reflecting treatment response.

Scalp HFO rates are not impacted by the skull defect resulting from craniotomy. Scalp HFO rates both within and outside the HFO area remained largely unaffected by the skull incision resulting from craniotomy, thus excluding a confounding effect of the skull defect on HFO detection and confirming the validity of comparisons between consecutive presurgical and postsurgical EEG recordings, as performed both in our previous, exploratory study ${ }^{14}$, and in our current, validating dataset. Furthermore, while scalp HFO rates in channels over the HFO area decreased following successful epilepsy surgery, irrespective of their proximity to the skull defect, scalp HFO rates in channels outside the HFO area but close to the skull defect did not increase following surgery. Our findings suggest that monitoring disease severity and response to treatment after craniotomy for epilepsy surgery is feasible and reliable since the resulting skull defect does not interfere with HFO detection. 
In the present study, we have shown for the first time that scalp HFO rates are not impacted by the skull defect resulting from craniotomy, including electrode contacts within close distance from the skull defect. This very reassuring finding concerning HFO in scalp EEG contrasts with several reports of skull defects modifying the appearance of spikes in scalp EEG to the extent that they can be mistaken for electrode artifacts and thus escape detection $^{40,41}$. In these reports, the scalp EEG recorded by a standard 10-20 electrode array illustrated cortically generated spikes as phase reversals without a physiological field due to the compact field configuration resulting from the skull defect. Furthermore, mu and beta rhythms may resemble spikes in the context of a pronounced breach rhythm ${ }^{42}$, thus adding to the risk of false positives in spike detection at the presence of a skull defect.

Beyond the detection and interpretation of scalp EEG signals in the HFO range in follow-up recordings after epilepsy surgery, as implemented in our study, our observations have crucial implications for patients undergoing presurgical investigation after failed epilepsy surgery or after a first resection guided by surgical indications, as in tumors and cavernomas. Considering that reoperations in pediatric epilepsy surgery are no rarity $2,3,5,43-45$, the fact that scalp HFO rates remain unaffected by the skull defect resulting from craniotomy is particularly encouraging for the use of scalp HFO both in the postsurgical setting, to guide crucial decisions such as the initiation of ASM withdrawal and in the presurgical setting, to guide the decision for epilepsy surgery and the delineation of surgical resection in selected candidates.

Future directions. Beyond the specific setting of epilepsy surgery, the correlation between HFO rates and seizure frequency may provide a valuable tool for monitoring disease severity and treatment success in this patient group, irrespective of the therapeutic approach. Novel and reliable non-invasive EEG biomarkers are urgently needed, particularly in pediatric epilepsy, since currently available biomarkers are insufficient to support treatment management. Spikes, the EEG biomarker currently in clinical use, lack specificity as they are sometimes recorded even in the absence of epileps $y^{46,47}$, while their rates correlate neither with seizure frequency nor with treatment response in the presence of epilepsy ${ }^{48}$. Considering that epilepsy incidence is higher in early life $^{49}$ and pediatric epilepsies often take a catastrophic course $\mathrm{s}^{2,43,50}$, novel biomarkers are likely to have the most significant impact in children and adolescents. A novel epilepsy biomarker that would efficiently monitor the disease state, thus guiding treatment choices, is poised to drastically change epilepsy management, possibly improving not only seizure but also cognitive outcomes in this vulnerable age group.

Despite the encouraging results of our study, scalp HFO need to be evaluated as a novel method against the current standards of prognostication in sufficiently large samples from multicentric studies. Furthermore, the validity and reliability of scalp HFO as an independent prognostic factor have to be assessed in multifactorial clinical prognosis scores allowing the integration of clinical, EEG, and imaging data and scalp HFO rates. In particular, conclusive data regarding the seizure recurrence risk after ASM withdrawal are required and the identification of relevant risk factors will have to outperform currently available predictive model ${ }^{51}$ with their inherent accuracy issues ${ }^{52}$ by integrating more informative diagnostic tools as covariates, such as neurophysiological markers of the epileptogenic network, including scalp HFO.

Finally, beyond the effect of skull defects resulting from craniotomy on the validity of postsurgical scalp HFO, as investigated in the present study, further factors should be addressed that may potentially affect postsurgical scalp HFO, such as the extent of craniotomy or the extent of resection. Although a larger craniotomy and resection may have a greater overall impact on scalp EEG signal, scalp HFOs have been shown to be generated by and synchronized over small brain regions ${ }^{18}$, thus adding to the ambiguity concerning the impact of craniotomy and resection extent on scalp HFO. This direction could be pursued in future datasets of pre- and postsurgical scalp EEG recordings from larger pediatric cohorts with drug-resistant focal lesional epilepsy undergoing resective epilepsy surgery that will serve to validate and expand the findings of the current study. In addition, larger datasets than the one analyzed in our study could potentially serve to develop statistically more robust modelling approaches, e.g. taking into account the correlation of within-subject data (multiple HFO rates and electrode distance measurements over time per channel within a given subject), thus refining our current conclusions.

\section{Conclusion}

We demonstrated that the decrease of scalp HFO rates over the HFO area correlated with long-term seizure freedom in our patients, even after the ASM had been withdrawn. In parallel, scalp HFO rates both within and outside the HFO area remained unaffected by the skull defect resulting from craniotomy, even in electrodes within close distance from the skull defect. Our study thus establishes the potential of scalp HFO for assessing treatment response in the pediatric population undergoing epilepsy surgery, potentially facilitating seizure outcome prognostication and thus guiding crucial therapeutic decisions, such as the initiation of ASM withdrawal.

\section{Data availability}

The raw EEG data and the HFO markings will be published upon acceptance of the article.

\section{Code availability}

The HFO detection software is freely available at the GitHub repository (https://github.com/ZurichNCH/Autom atic-High-Frequency-Oscillation-Detector).

Received: 21 October 2021; Accepted: 5 January 2022

Published online: 25 January 2022

\section{References}

1. Dwivedi, R. et al. Surgery for drug-resistant epilepsy in children. N. Engl. J. Med. 377, 1639-1647 (2017). 
2. Kadish, N. E. et al. Epilepsy surgery in the first 3 years of life: Predictors of seizure freedom and cognitive development. Neurosurgery 84, E368-E377 (2019).

3. Kogias, E. et al. Multilobar epilepsy surgery in childhood and adolescence: Predictors of long-term seizure freedom. Neurosurgery 88, 174-182 (2020).

4. Ramantani, G. et al. Epilepsy surgery for glioneuronal tumors in childhood: avoid loss of time. Neurosurgery 74, 648-657; discussion 657 (2014).

5. Ramantani, G. et al. Frontal lobe epilepsy surgery in childhood and adolescence: Predictors of long-term seizure freedom. Overall cognitive and adaptive functioning. Neurosurgery 83, 93-103 (2018).

6. Ramantani, G. et al. Posterior cortex epilepsy surgery in childhood and adolescence: Predictors of long-term seizure outcome. Epilepsia 58, 412-419 (2017).

7. Lamberink, H. J. et al. Changing profiles of pediatric epilepsy surgery candidates over time: A nationwide single-center experience from 1990 to 2011. Epilepsia 56, 717-725 (2015).

8. Barba, C. et al. Trends in pediatric epilepsy surgery in Europe between 2008 and 2015: Country-, center-, and age-specific variation. Epilepsia 61, 216-227 (2020).

9. Maillard, L. G. et al. Stereoelectroencephalography and surgical outcome in polymicrogyria-related epilepsy: A multicentric study. Ann. Neurol. 82, 781-794 (2017).

10. Boshuisen, K. et al. Intelligence quotient improves after antiepileptic drug withdrawal following pediatric epilepsy surgery. Ann. Neurol. 78, 104-114 (2015).

11. Lamberink, H. J. et al. Why the TimeToStop trial failed to recruit: A survey on antiepileptic drug withdrawal after paediatric epilepsy surgery. Epileptic Disord. 20, 374-385 (2018).

12. Ramantani, G. \& Reuner, G. Cognitive development in pediatric epilepsy surgery. Neuropediatrics 49, 93-103 (2018).

13. Jacobs, J. \& Zijlmans, M. HFO to measure seizure propensity and improve prognostication in patients with epilepsy. Epilepsy Curr. 1535759720957308 (2020). https://doi.org/10.1177/1535759720957308.

14. Boran, E., Sarnthein, J., Krayenbühl, N., Ramantani, G. \& Fedele, T. High-frequency oscillations in scalp EEG mirror seizure frequency in pediatric focal epilepsy. Sci. Rep. 9, 16560 (2019).

15. Melani, F., Zelmann, R., Dubeau, F. \& Gotman, J. Occurrence of scalp-fast oscillations among patients with different spiking rate and their role as epileptogenicity marker. Epilepsy Res. 106, 345-356 (2013).

16. Andrade-Valenca, L. P., Dubeau, F., Mari, F., Zelmann, R. \& Gotman, J. Interictal scalp fast oscillations as a marker of the seizure onset zone. Neurology 77, 524-531 (2011).

17. Cai, Z. et al. Noninvasive high-frequency oscillations riding spikes delineates epileptogenic sources. Proc. Natl. Acad. Sci. USA 118, (2021).

18. Zelmann, R., Lina, J. M., Schulze-Bonhage, A., Gotman, J. \& Jacobs, J. Scalp EEG is not a blur: It can see high frequency oscillations although their generators are small. Brain Topogr. 27, 683-704 (2014).

19. Pizzo, F. et al. Detectability of fast ripples $(>250 \mathrm{~Hz}$ ) on the scalp EEG: A proof-of-principle study with subdermal electrodes. Brain Topogr. 29, 358-367 (2016).

20. von Ellenrieder, N., Frauscher, B., Dubeau, F. \& Gotman, J. Interaction with slow waves during sleep improves discrimination of physiologic and pathologic high-frequency oscillations (80-500 Hz). Epilepsia 57, 869-878 (2016).

21. van Klink, N. et al. Beamforming applied to surface EEG improves ripple visibility. Clin. Neurophysiol. 129, 101-111 (2018).

22. Delucchi, M. R., Garoutte, B. \& Aird, R. B. The scalp as an electroencephalographic averager. Electroencephalogr. Clin. Neurophysiol. 14, 191-196 (1962).

23. Cobb, W. \& Sears, T. A. A study of the transmission of potentials after hemispherectomy. Electroencephalogr. Clin. Neurophysiol. 12, 371-383 (1960).

24. Cobb, W. A., Guiloff, R. J. \& Cast, J. Breach rhythm: The EEG related to skull defects. Electroencephalogr. Clin. Neurophysiol. 47, 251-271 (1979).

25. Brigo, F., Cicero, R., Fiaschi, A. \& Bongiovanni, L. G. The breach rhythm. Clin. Neurophysiol. 122, 2116-2120 (2011).

26. Voytek, B. et al. Hemicraniectomy: A new model for human electrophysiology with high spatio-temporal resolution. J. Cogn. Neurosci. 22, 2491-2502 (2010).

27. Engel J Jr, Van Ness PC, Rasmussen TB, \& Ojemann LM. Outcome with respect to epileptic seizures. in Surgical treatment of the epilepsies (ed. Engel J Jr) 609-21 (Raven Press, 1993).

28. Berry, R. B. et al. AASM scoring manual updates for 2017 (Version 2.4). J Clin. Sleep Med. 13, 665-666 (2017).

29. Cserpan, D. et al. Scalp high-frequency oscillation rates are higher in younger children. Brain Commun. https://doi.org/10.1093/ braincomms/fcab052 (2021).

30. Burnos, S. et al. Human intracranial high frequency oscillations (HFOs) detected by automatic time-frequency analysis. PLoS ONE 9, e94381 (2014).

31. Fedele, T. et al. Resection of high frequency oscillations predicts seizure outcome in the individual patient. Sci. Rep. 7, 13836 (2017).

32. Fedele, T. et al. Automatic detection of high frequency oscillations during epilepsy surgery predicts seizure outcome. Clin. Neurophysiol. 127, 3066-3074 (2016).

33. von Ellenrieder, N., Beltrachini, L., Perucca, P. \& Gotman, J. Size of cortical generators of epileptic interictal events and visibility on scalp EEG. Neuroimage 94, 47-54 (2014).

34. Kuhnke, N., Klus, C., Dümpelmann, M., Schulze-Bonhage, A. \& Jacobs, J. Simultaneously recorded intracranial and scalp high frequency oscillations help identify patients with poor postsurgical seizure outcome. Clin. Neurophysiol. 130, 128-137 (2019).

35. Cuello-Oderiz, C., von Ellenrieder, N., Dubeau, F. \& Gotman, J. Influence of the location and type of epileptogenic lesion on scalp interictal epileptiform discharges and high-frequency oscillations. Epilepsia https://doi.org/10.1111/epi.13922 (2017).

36. Kobayashi, K., Akiyama, T., Oka, M., Endoh, F. \& Yoshinaga, H. A storm of fast (40-150Hz) oscillations during hypsarrhythmia in West syndrome. Ann. Neurol. 77, 58-67 (2015).

37. Gong, P. et al. Scalp-recorded high-frequency oscillations in childhood epileptic encephalopathy with continuous spike-and-wave during sleep with different etiologies. Brain Dev. 40, 299-310 (2018).

38. Qian, P., Li, H., Xue, J. \& Yang, Z. Scalp-recorded high-frequency oscillations in atypical benign partial epilepsy. Clin. Neurophysiol. 127, 3306-3313 (2016).

39. Cao, D. et al. Scalp EEG high frequency oscillations as a biomarker of treatment response in epileptic encephalopathy with continuous spike-and-wave during sleep (CSWS). Seizure 71, 151-157 (2019).

40. Mader, E. C., Miller, D., Toler, J. M. \& Olejniczak, P. W. Focal epileptiform discharges can mimic electrode artifacts when recorded on the scalp near a skull defect. J. Investig. Med. High Impact. Case Rep. 6, 2324709618795305 (2018).

41. Moores, M., Picheca, C. \& Fantaneanu, T. A. Electropositive seizures and non-convulsive status epilepticus in a critically ill patient with prior skull defect. Epileptic Disord. 22, 219-223 (2020).

42. Radhakrishnan, K., Chandy, D., Menon, G. \& Sarma, S. Clinical and electroencephalographic correlates of breach activity. Am. J. Electroneurodiagn. Technol. 1, 138-147 (1999).

43. Ramantani, G. et al. Seizure and cognitive outcomes of epilepsy surgery in infancy and early childhood. Eur. J. Paediatr. Neurol. 17, 498-506 (2013).

44. Ramantani, G. et al. Reoperation for refractory epilepsy in childhood: A second chance for selected patients. Neurosurgery 73 , 695-704 (2013). 
45. Ramantani, G. et al. Seizure control and developmental trajectories after hemispherotomy for refractory epilepsy in childhood and adolescence. Epilepsia 54, 1046-1055 (2013).

46. Cavazzuti, G. B., Cappella, L. \& Nalin, A. Longitudinal study of epileptiform EEG patterns in normal children. Epilepsia 21, 43-55 (1980).

47. So, E. L. Interictal epileptiform discharges in persons without a history of seizures: What do they mean?. J. Clin. Neurophysiol. 27, 229-238 (2010).

48. Goncharova, I. I. et al. The relationship between seizures, interictal spikes and antiepileptic drugs. Clin. Neurophysiol. 127, 31803186 (2016)

49. Kotsopoulos, I. A. W., van Merode, T., Kessels, F. G. H., de Krom, M. C. T. F. M. \& Knottnerus, J. A. Systematic review and metaanalysis of incidence studies of epilepsy and unprovoked seizures. Epilepsia 43, 1402-1409 (2002).

50. Ramantani, G. Epilepsy surgery in early life: The earlier the better. World Neurosurg. 131, 285-286 (2019).

51. Lamberink, H. J. et al. Individualised prediction model of seizure recurrence and long-term outcomes after withdrawal of antiepileptic drugs in seizure-free patients: A systematic review and individual participant data meta-analysis. Lancet Neurol. 16, 523-531 (2017).

52. Contento, M. et al. Prediction of seizure recurrence risk following discontinuation of antiepileptic drugs. Epilepsia 62, 2159-2170 (2021).

\section{Acknowledgements}

We thank the neurophysiology technicians B. Alessandri, C. Carosio, P. Hieber, and G. Selmin for their assistance with EEG recordings and data analysis. We thank the Swiss National Science Foundation (CRSK-3_190895 to G.R. and J.S.), and the Anna Mueller Grocholski Foundation (to G.R.) for funding. The funders had no role in the design or analysis of the study.

\section{Author contributions}

D.C., A.G., and L.G. analyzed data. D.C. and G.R. prepared figures and tables. G.R. treated patients and monitored outcome. SP.LB. acquired data. G.R. designed and supervised the study. D.C., A.G, R.T, J.S. and G.R. wrote the article. All authors critically reviewed the manuscript.

\section{Funding}

This article was funded by Swiss National Science Foundation (Grant no. CRSK-3_190895) and Anna Mueller Grocholski Foundation.

\section{Competing interests}

The authors declare no competing interests.

\section{Additional information}

Supplementary Information The online version contains supplementary material available at https://doi.org/ 10.1038/s41598-022-05373-x.

Correspondence and requests for materials should be addressed to G.R.

Reprints and permissions information is available at www.nature.com/reprints.

Publisher's note Springer Nature remains neutral with regard to jurisdictional claims in published maps and institutional affiliations.

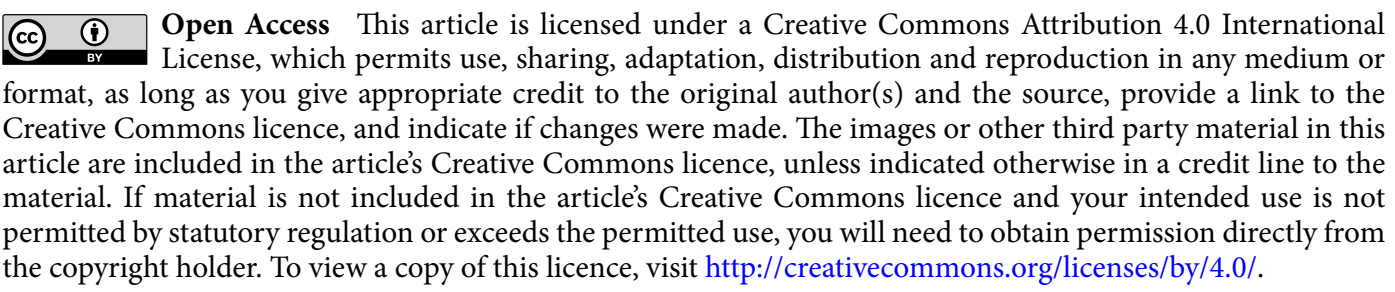

(C) The Author(s) 2022 